\title{
28 Research Square \\ IGF-2 modified by the m6A demethylation enzyme ALKBH5 in the ossification of the ligamentum flavum
}

\author{
Ming-jie Kuang \\ Shandong Provincial Hospital \\ Hai-feng Wang \\ Shandong Provincial Hospital \\ Jie Qiu \\ Shandong University \\ An-bang Wang \\ Shandong University \\ Feng Wang \\ Shandong Provincial Hospital \\ Bing-yi Tan \\ Shandong Provincial Hospital \\ Shi-jie Han \\ Shandong Provincial Hospital \\ Xiao-ming Li \\ Cangzhou People's Hospital \\ Da-Chuan Wang ( $\sim$ wangdachuansd@163.com ) \\ Shandong Provincial Hospital
}

\section{Research}

Keywords: RNA methylation, Ossification of the ligamentum flavu, m6A, IGF-2区ALKBH5

Posted Date: April 13th, 2021

DOl: https://doi.org/10.21203/rs.3.rs-399010/v1

License: (c) (1) This work is licensed under a Creative Commons Attribution 4.0 International License.

Read Full License 


\section{Abstract}

Abstract Background: Ossification of the ligamentum flavum (OLF) is a pathological heterotopic ossification of the paravertebral ligament. However, the specific pathophysiology mechanism of this disease is still unknown. The $\mathrm{m} 6 \mathrm{~A}$ methylation and its potential functions in OLF remain to be unexplored. Method: In this study, we performed a transcriptome-wide methylation analysis using the OLF and normal ligaments to explore the mechanism of OLF. Common and region-specific methylation have different preferences for methylation site selection and thereby different impacts on their biological functions. We screened out the methylase-ALKBH5, and by promoting or inhibiting its expression, observing the content of $\mathrm{m} 6 \mathrm{~A}$, and measuring the ossification of ligaments, and then measuring IGF expression situation by alizarin red staining, alkaline phosphatase, immunofluorescence, immunohistochemistry, etc. Result: MeRIP-seq and qPCR showed that the m6A methylation level of OLF group was usually higher than that of control group. In addition, we found that ALKBH5 is an important demethyltransferase in OLF, which promotes the expression of $\mathrm{m} 6 \mathrm{~A}$. ALKBH5 promotes the expression of IGF-2, which in turn promotes osteogenesis in OLF. Conclusion: Overall, we provided a region-specific map of $\mathrm{m} 6 \mathrm{~A}$ methylation and characterized the distinct features of specific and common methylation in OLF, and we proved that IGF-2 can be regulated by ALKBH5 to promote the process of OLF.

\section{Full Text}

This preprint is available for download as a PDF.

\section{Figures}


A

OLF

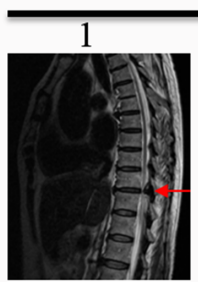

MRI

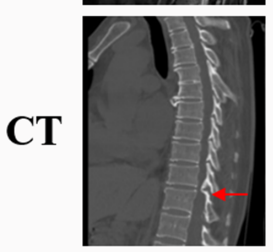

B

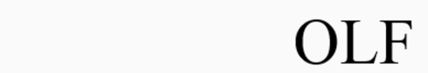

HE

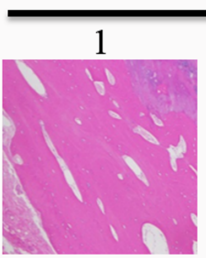

Control

C

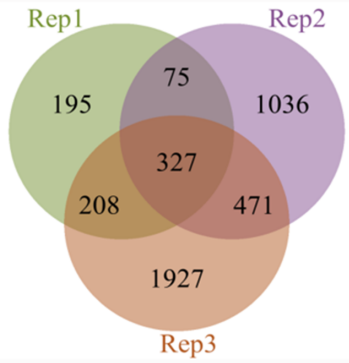

F

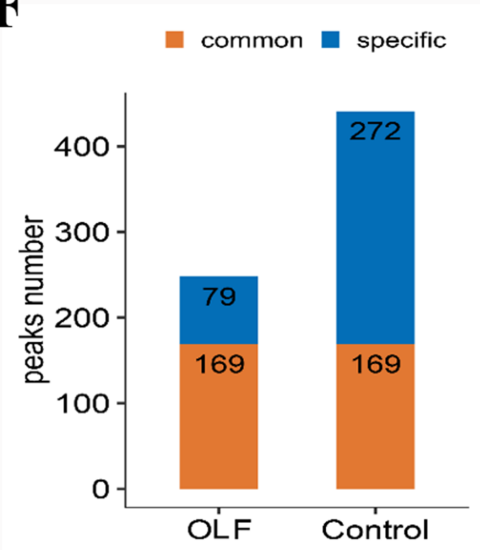

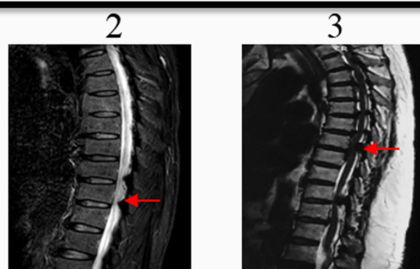

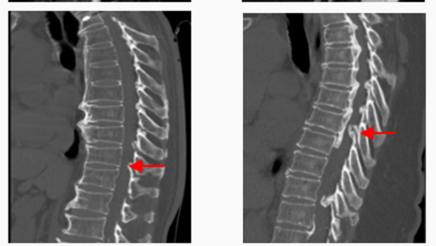

2
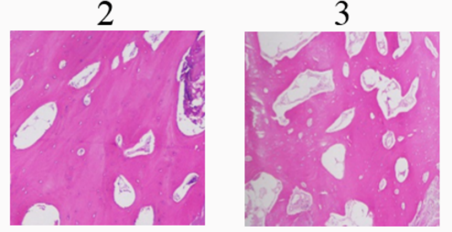

OLF

D

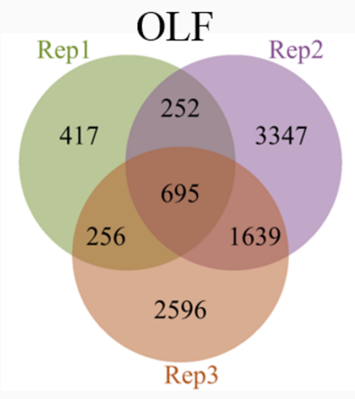

G

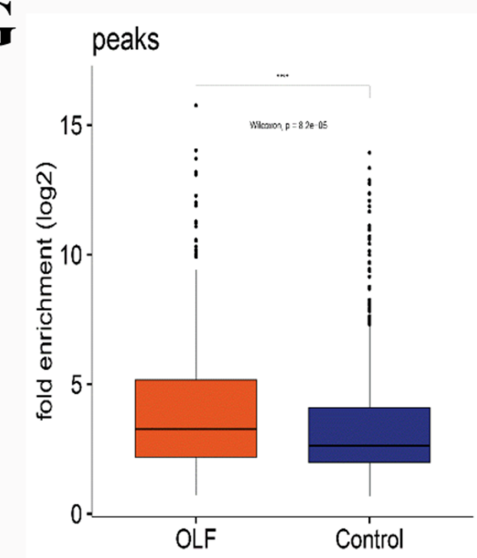

Control
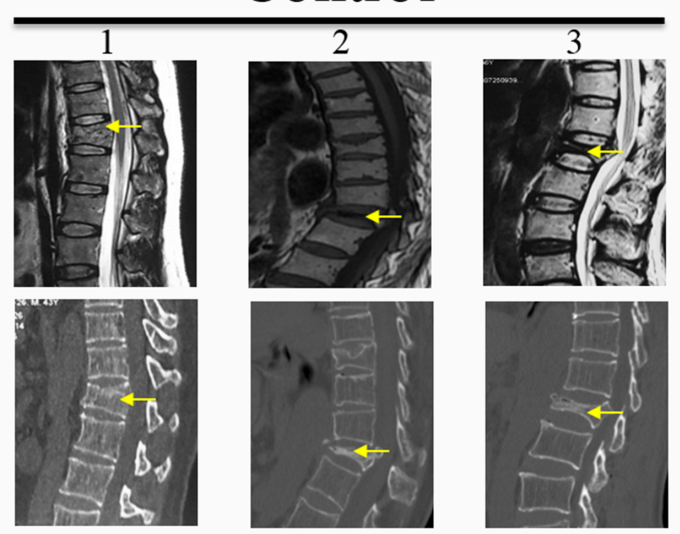

Control

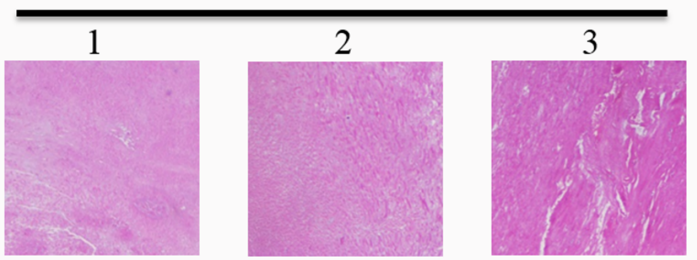

$\mathbf{E}$

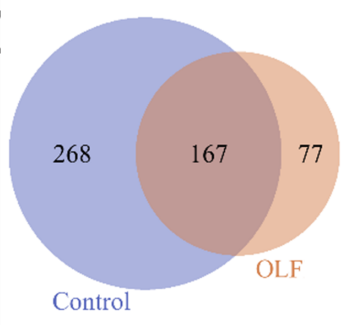

H

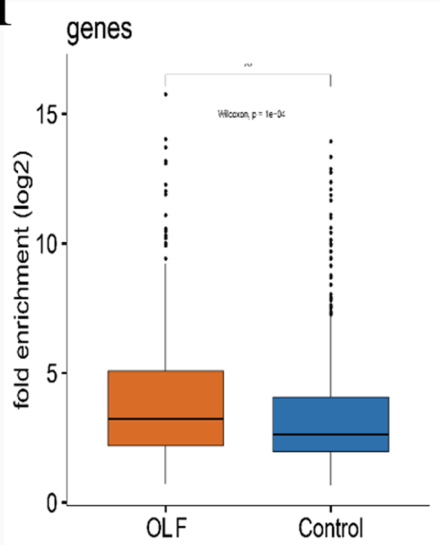

\section{Figure 1}

The identification of OLF and control tissues in morphology and m6A methylation. (A) The MRI and CT scan of OLF and fracture patients to identify the ossification of ligamentum flavum. (B) The HE staining was performed to detect the morphology of OLF and normal ligamentum flavum. (C, D) Venn diagrams was conducted to identify the numbers of overlapping m6A transcripts in the two biological replicates of m6A-IP the control and the OLF groups. (E) Venn diagram showing the numbers of CMRs and SMRs 
between the OLF and control group. (F) Column chart showed the numbers of common and specific m6A peaks between the OLF and control groups. The blue bars indicated common peaks, while the orange bars indicated specific peaks. $(G, H)$ Box plots was conducted to present the methylation levels of OLF RNAs and non-OLF RNAs by comparing the median fold enrichment at peak levels (G) and gene levels $(\mathrm{H})$.

A

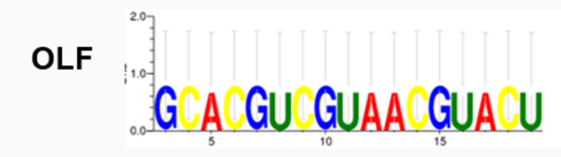

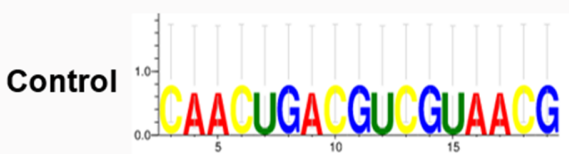

D

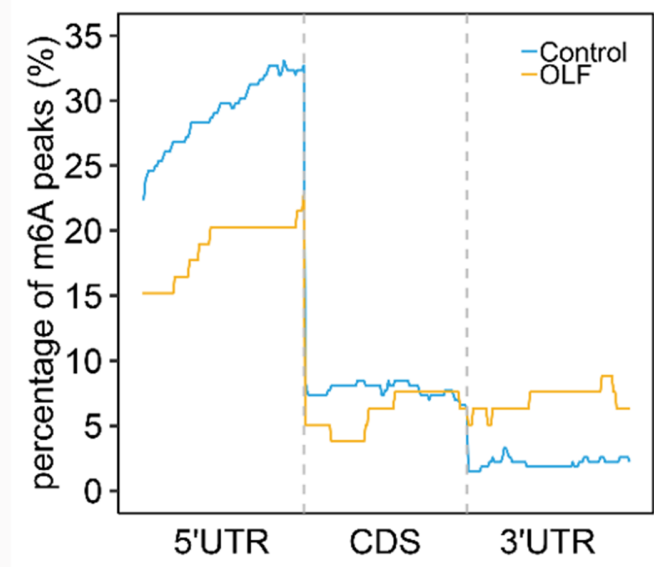

$\mathbf{F}$

genes

早 CMR 自 SMR

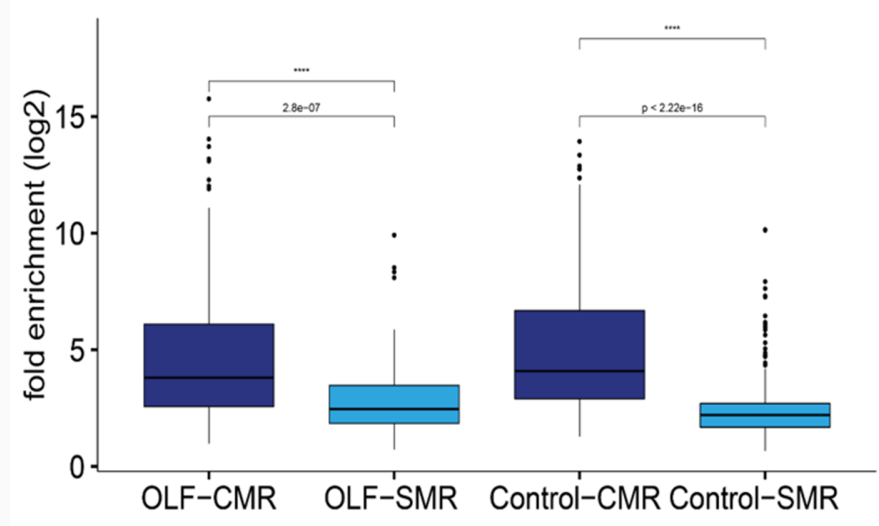

All peaks

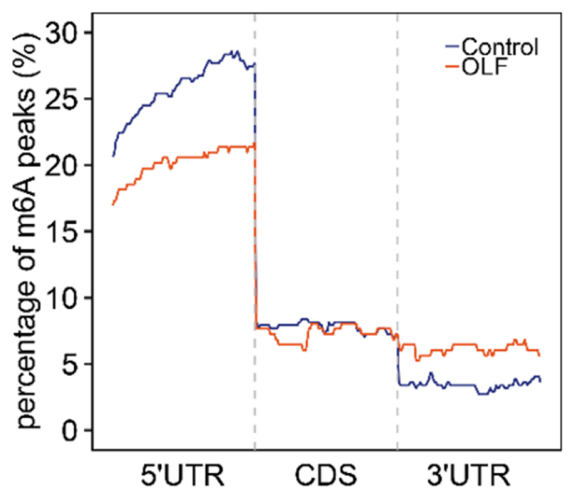

C Common peaks

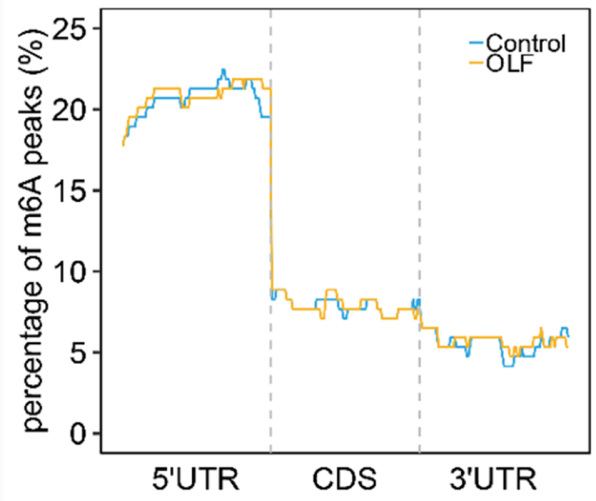

E
Specific peaks

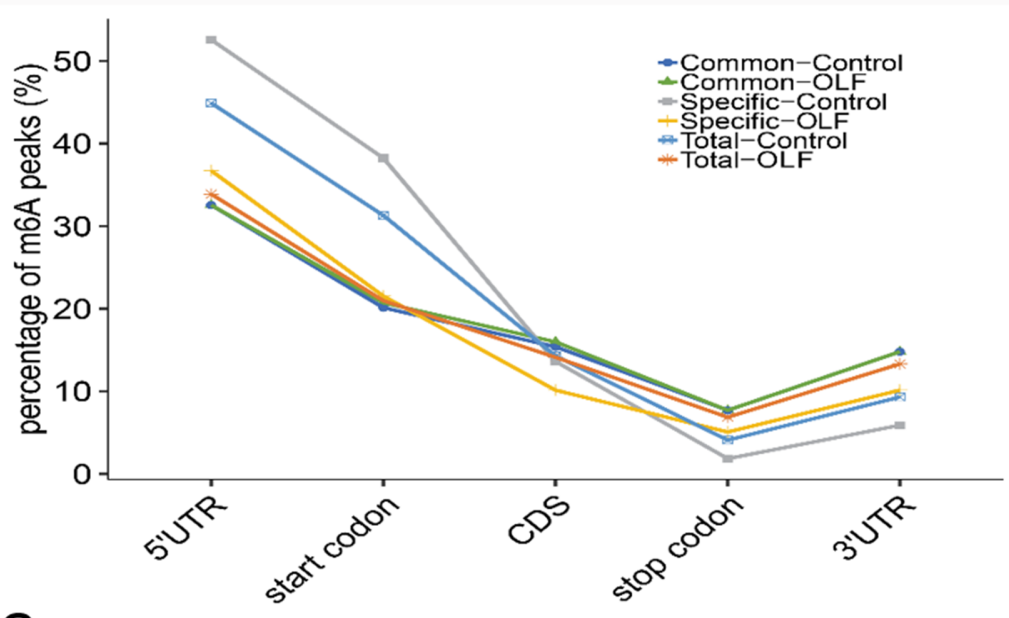

peaks

自 $C M R$ 自 $S M R$

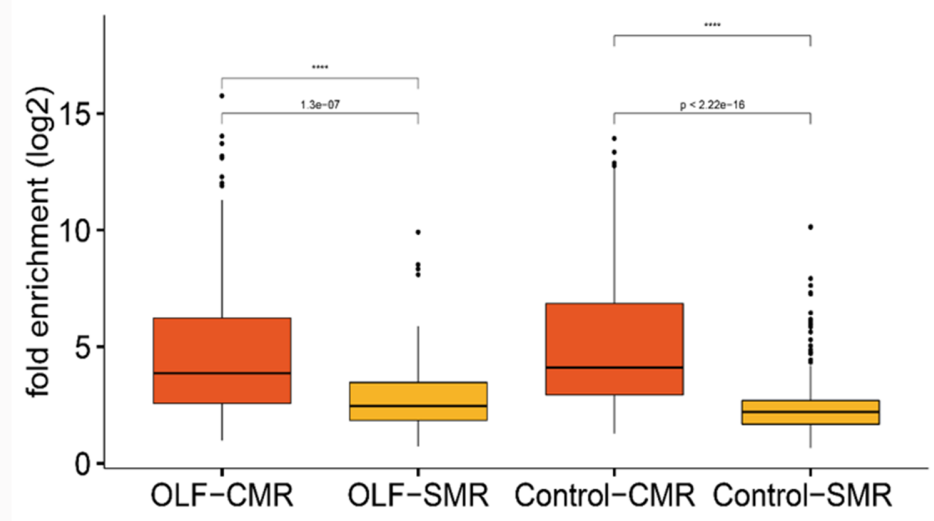

Figure 2 
Distribution patterns of m6A peaks between the OLF and control group were detected using MeRIP-seq. (A) Sequence logo representing the deduced consensus motif through clustering of all enriched m6A peaks between OLF and control group. (B-D) Enrichment of all m6A peaks (B), common m6A peaks (C) and specific m6A peaks (D) along the whole mRNA transcripts. (E) The percentage of specific m6A peaks along the 5'UTR, start codon, CDS, stop codon and 3'UTR between common-control group, common-OLF group, specific-control group, specific-OLF group and total-OLF. $(F, G)$ Box plots was conducted to detect the methylation levels of CMRs and SMRs by comparing the fold enrichment at the gene level $(F)$ or peaks level (G). Wilcoxon test was performed for statistical analysis. ${ }^{* \star} p$ value $<0.001$.

A

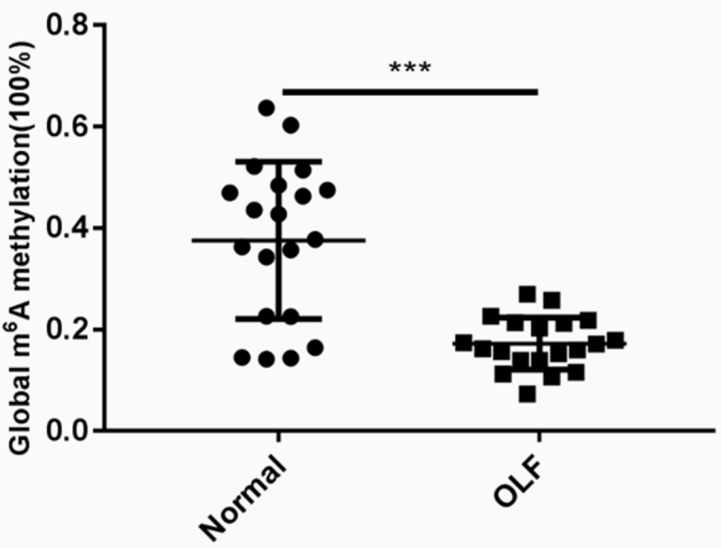

C
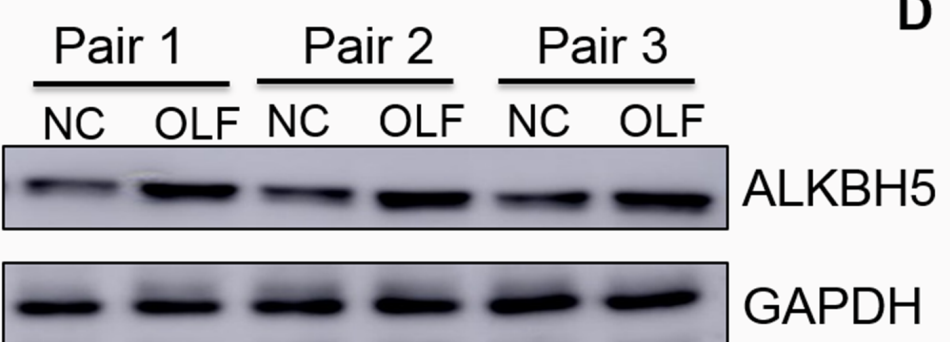

GAPDH

E
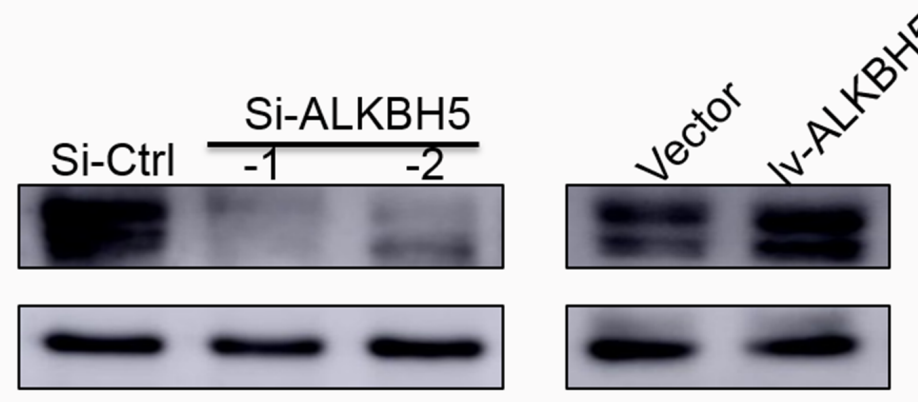

ALKBH5
GAPDH
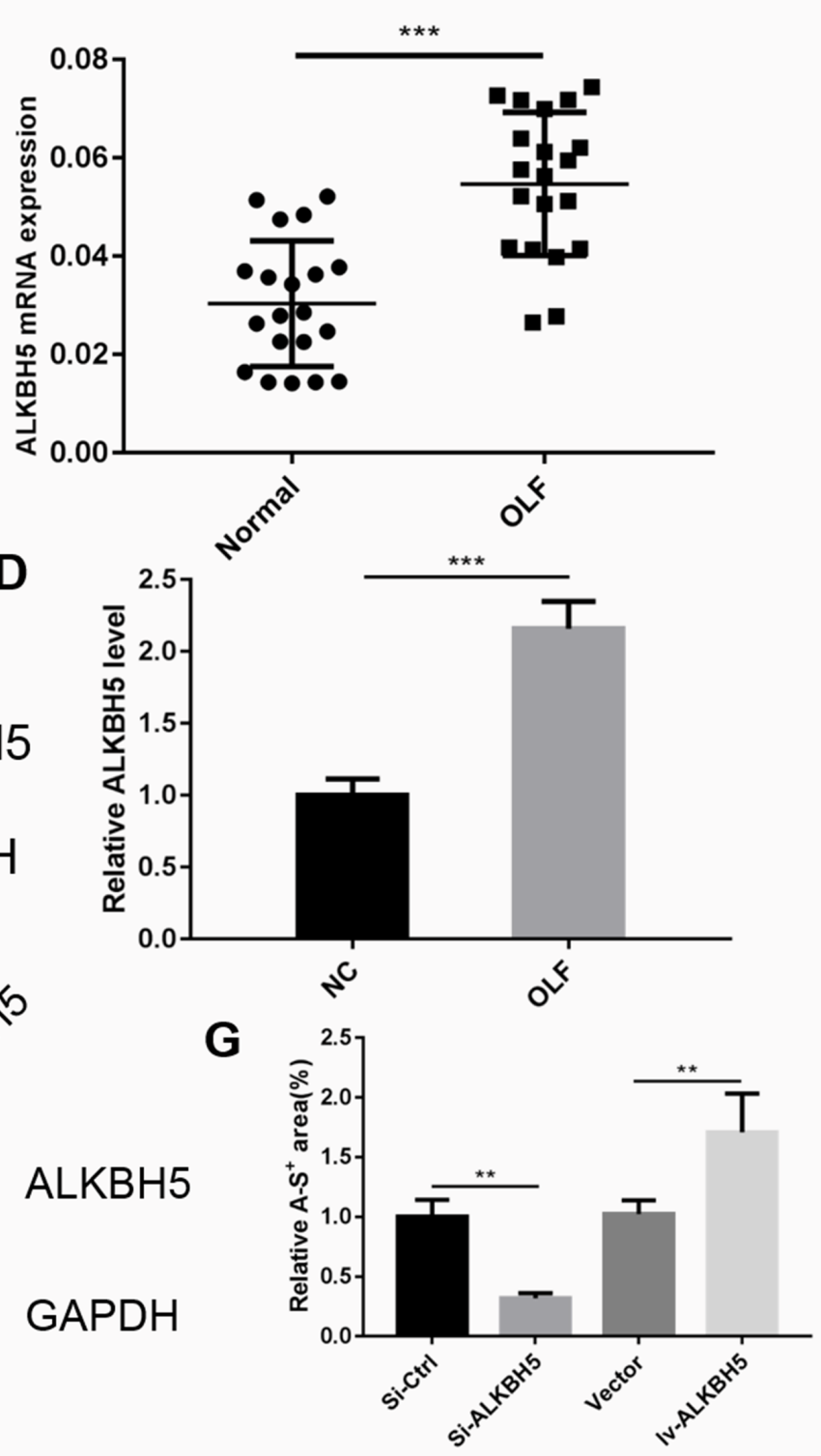

F

Si-Ctrl

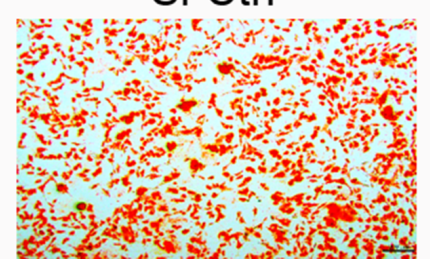

Si-ALKBH5

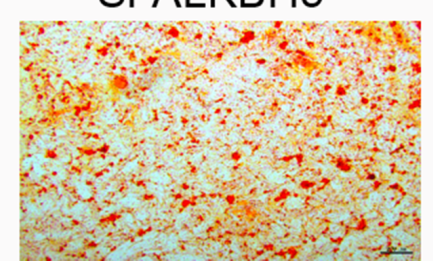

Vector

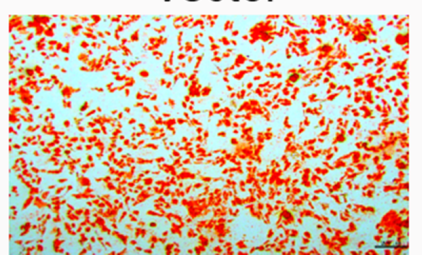

IV-ALKBH5

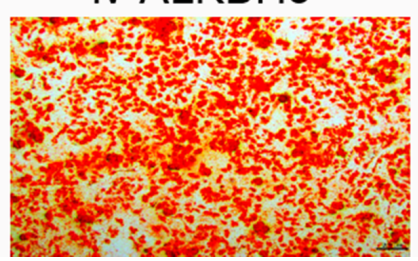

Figure 3 
The m6A methylation was detected in OLF or control groups. (A) The global of m6A methylation was detected between OLF and normal group. (B) The expression of ALKBH5 was detected using q-PCR between OLF and normal groups. (C) Western blotting was performed to verify the expression of ALKBH5 in three pairs of tissues. (D) The quantitative calculation of western blotting using Image $\mathrm{J}$ software. (E) The transfection efficiency of knock down and overexpression of ALKBH5 was evaluated using western blotting. (F) The alizarin red staining was performed to investigate the osteogenesis when transfected with si-ALKBH5 or IVALKBH5. (G) The quantitative calculation of alizarin red staining using Image $J$ software.

A

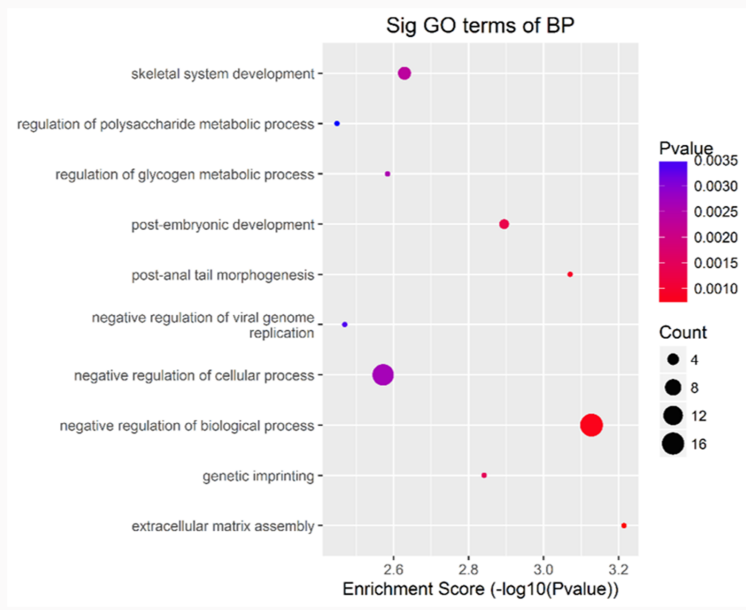

C

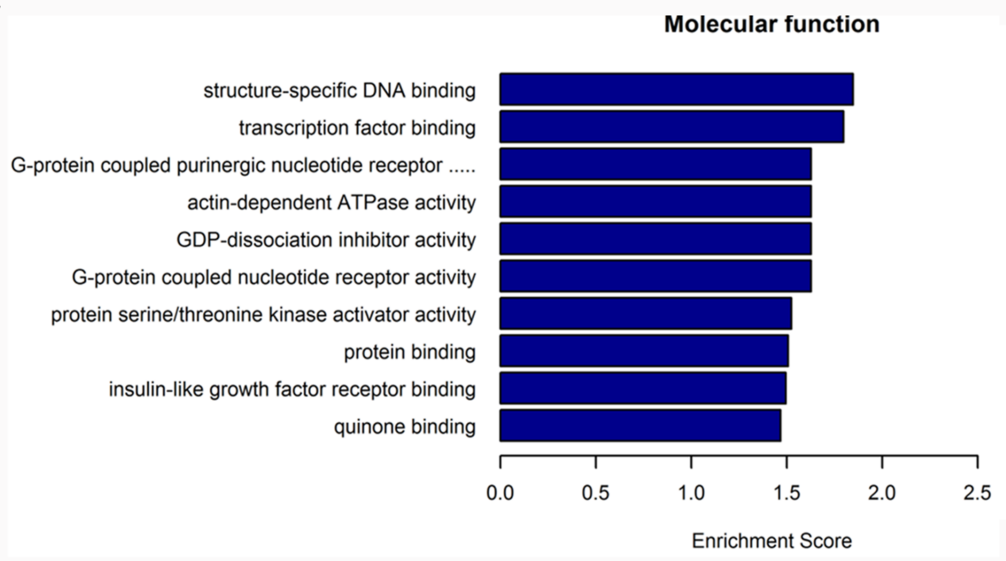

B

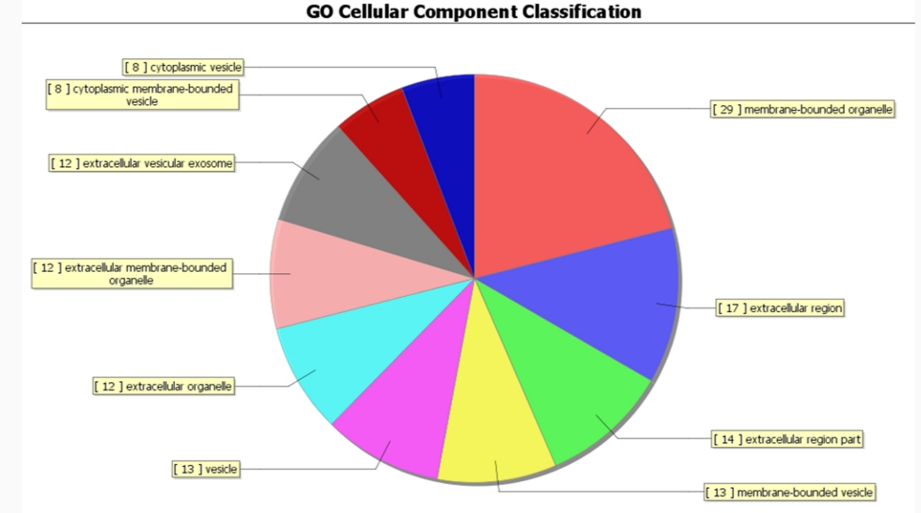

D
E

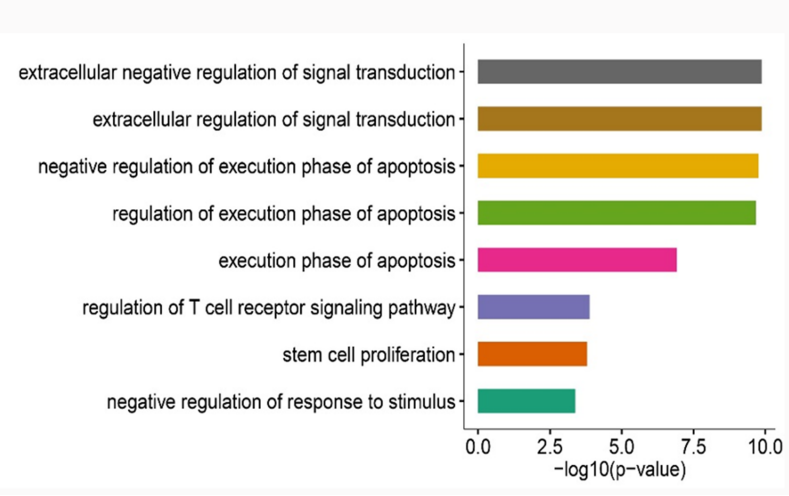

F

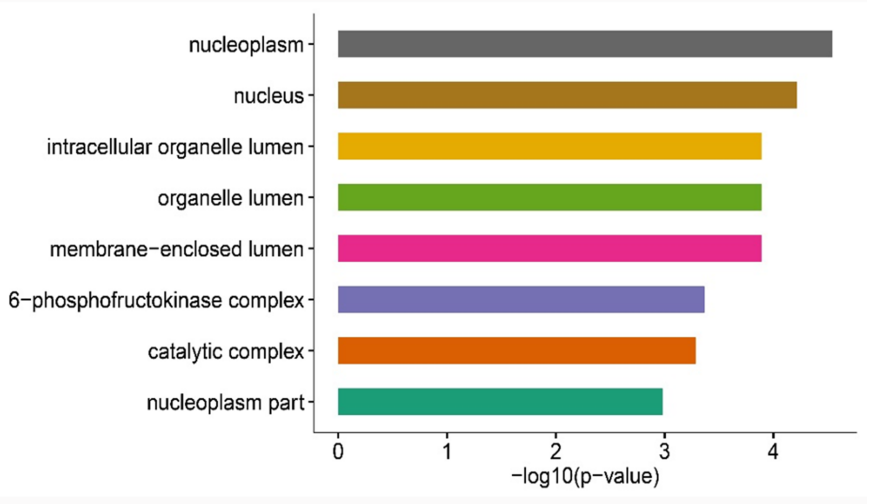

Figure 4 
Gene ontology analysis of commonly and specifically methylated genes was conducted between the OLF and control groups. (A) The significant $\mathrm{GO}$ terms of BP was presented using bubble diagram. (B) GO cellular component of classification was investigated using pie chart. (C) The molecular function analysis of was performed to investigate the potential mechanism of m6A in OLF. (D-F) GO functional analysis of specifically methylated genes was performed to detect the related molecular mechanism of m6A methylation in OLF.
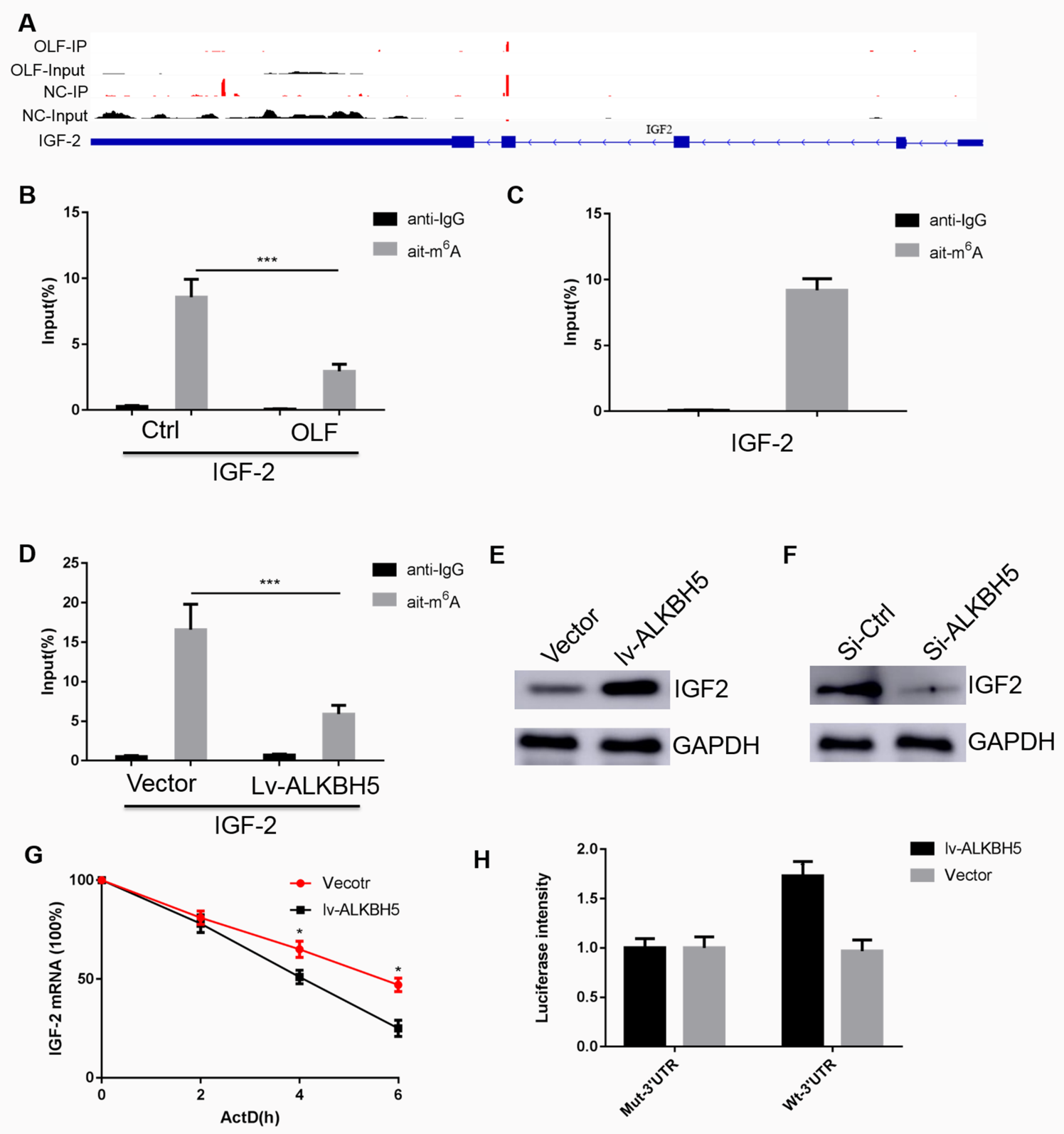

H
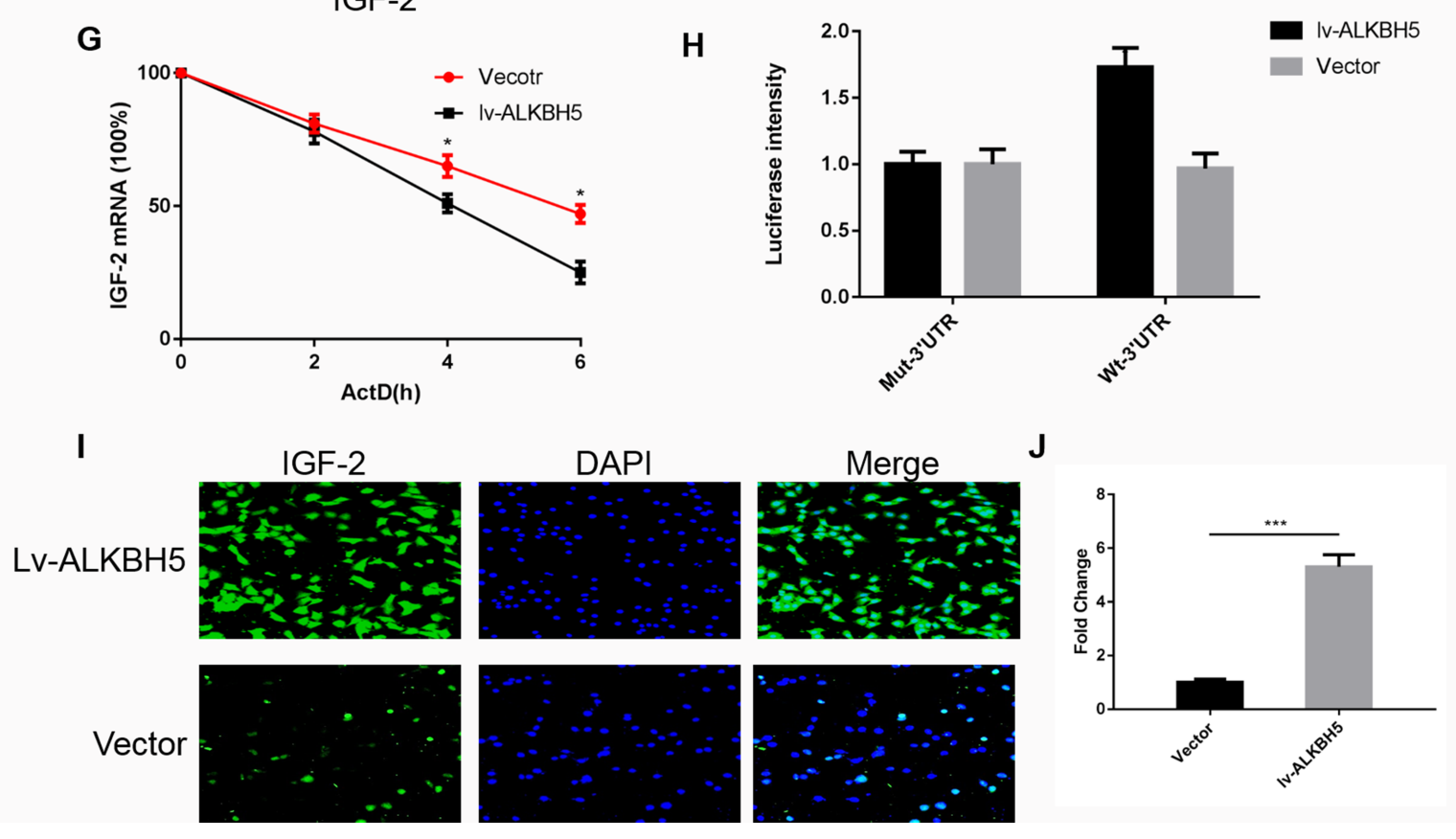

Figure 5 
The m6A methylation of IGF-2 in OLF was detected. (A) The m6A methylation of IGF-2 was visualized using MeRIP-seq between the IP and Input group. (B) MeRIP-qPCR was performed to investigate the m6A methylation of IGF-2 between the OLF and control group. (C) The m6A methylation of IGF-2 was detected in normal ligamentum flavu cells. (D) The m6A methylation of IGF-2 was detected when transfected with ALKBH5 overexpression. (E) The expression of IGF-2 was investigated with ALKBH5 overexpression. (F) The expression of IGF-2 was investigated with ALKBH5 knock down. (G) The stability of IGF-2 mRNA was detected using ActD assay. (H) The 3'-UTR of IGF-2 (wild-type and mutant) was cloned and luciferase reporter assay was performed, and these construct was co-transfected with the Iv-ALKBH5 or vector into ligamentum flavu cells. (I) Immunofluorescence staining was performed to detect the expression of IGF-2 when transfected with ALKBH5 or vector. $(\mathrm{J})$ The quantitative calculation of immunofluorescence staining using Image $\mathrm{J}$ software.

A
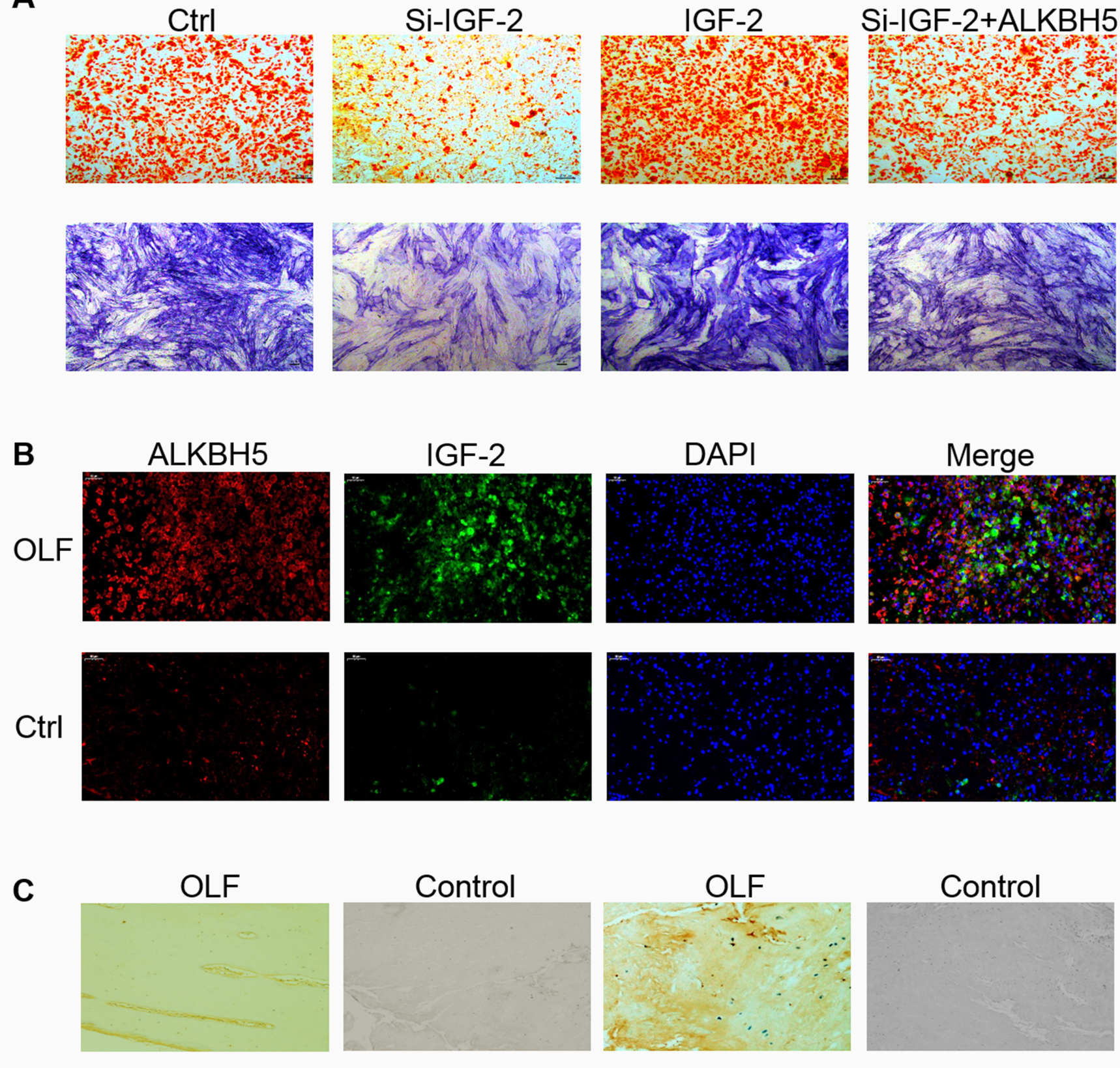

Control 
Figure 6

The expression of ALKBH5 and IGF-2 was verified in cell and tissues. (A) The alizarin red staining was performed to investigate the osteogenesis when transfected with si-IGF-2 or si-IGF-2+ALKBH5. (B) The expression of ALKBH5 and IGF-2 was evaluated in OLF and normal tissues. (C) IHC was performed to evaluate the expression of ALKBH5 and IGF-2.

\section{Supplementary Files}

This is a list of supplementary files associated with this preprint. Click to download.

- coverletter.pdf

- titlepage.docx 